\title{
Riscos da alimentação com leite artificial
}

Mónica Pina,* Carlo Volpato**

\section{RESUMO}

Neste artigo fez-se uma revisão dos riscos associados à alimentação das crianças com leite artificial, nomeadamente dos riscos para a criança, para a mãe, para o ambiente e para a sociedade. Na criança está aumentado o risco, entre outros, de mortalidade, gastroenterite aguda, otite, infecção respiratória baixa, asma e atopia, doença celíaca, diabetes mellitus tipo 1, doenças inflamatórias do intestino, leucemias agudas e linfomas, obesidade, diabetes mellitus tipo 2, hipertensão arterial e colesterol, morte súbita do lactente e má-oclusão dentária. Na mãe está aumentado, entre outros, o risco de cancro da mama, cancro do ovário, obesidade, enfarte agudo do miocárdio, diabetes mellitus tipo 2, depressão pós-parto, doença da vesícula biliar e osteoporose. Para o ambiente, alimentar com leite artificial aumenta o consumo de recursos que começam a escassear, e a acumulação de lixo não-biodegradável. Para a sociedade, há repercussões nos orçamentos familiar e do estado e consequências relacionadas com a alteração da vinculação mãe-bebé.

Procurou-se com este artigo reforçar a importância da promoção, protecção e apoio do aleitamento materno.

Palavras-Chave: Aleitamento Materno; Leite Artificial; Risco.

\section{INTRODUÇÃO}

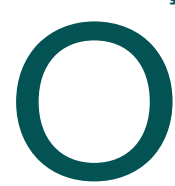

É hoje reconhecida a importância do aleitamento materno. Esse reconhecimento é de tal forma consensual que a Comissão Europeia criou, em 2004, um grupo que envolveu os Estados-membros, para que definisse estratégias para promover, proteger e apoiar o aleitamento materno na Europa. Do trabalho desse grupo resultou um documento onde se pode ler: «A protecção, promoção e apoio ao aleitamento materno são uma prioridade no que diz respeito à saúde pública em toda a Europa. Taxas baixas de aleitamento materno ou a sua cessação precoce têm implicações desfavoráveis importantes para a saúde e estrutura social da mulher, da criança, da comunidade e do meio ambiente; além disso, resultam num aumento das despesas do Sistema Nacional de Saúde, bem como no aumento das desigualdades na saúde». ${ }^{1}$

No sentido de ajudar os profissionais de saúde na promoção, protecção e apoio do aleitamento materno e de uniformizar as práticas nos vários países, foram

* Assistente Hospitalar de Medicina Interna no Hospital Nossa Senhora do Rosário (Barreiro), Conselheira em Aleitamento Materno, Moderadora da La Leche League

** Psicólogo, Psicoterapeuta, Clínica Smet, Lisboa. elaboradas recomendações para a alimentação infantil (Infant and young child feeding: standard recommendations for the European Union), que se encontram disponíveis na Internet. ${ }^{2}$

Também a American Academy of Pediatrics publicou, em 2005, um documento com o nome "Breastfeeding and the Use of Human Milk», onde se define o leite materno como «o alimento que garante ao lactente $o$ melhor estado possível de saúde física, o melhor desenvolvimento evolutivo e psicossocial?. Lê-se, ainda, que «o leite materno é espécie-específico, todos os preparados alimentares que o substituem são muito diferentes deste, o que torna o leite humano o alimento único e superior para a alimentação do lactente». ${ }^{3}$

De facto, as recomendações internacionais são consensuais em indicar 6 meses de amamentação exclusiva (o que quer dizer mesmo só leite materno, excepto em raros casos de risco de desidratação, em que a água pode estar recomendada), e a Organização Mundial da Saúde (OMS) e a United Nations Children's Fund (UNI$\mathrm{CEF)} \mathrm{recomendam} \mathrm{prolongar} \mathrm{a} \mathrm{amamentação,} \mathrm{comple-}$ mentando-a com alimentos adequados, até aos 2 anos, ou mais, se a mãe e o bebé assim o desejarem. ${ }^{4}$

Quando se fala e escreve sobre aleitamento materno, é comum fazer referência às suas «vantagens» ou 
«benefícios». Na nossa sociedade, a normalidade é a alimentação artificial e amamentar é a excepção. No entanto, a amamentação é a forma naturalmente adequada para alimentar as crianças, e deveria ser a norma. Seria, assim, mais correcto, falar-se de riscos da alimentação com leite artificial.

De facto, no Código Internacional de Ética do Marketing dos Substitutos do Leite Materno, no $4^{\circ}$ artigo, lê-se que os pais devem ser informados «do perigo para a saúde resultante do consumo desnecessário ou inadequado da fórmula para lactentes ou outros substitutos do leite materno». ${ }^{5}$

No que diz respeito à alimentação com leite artificial, podemos distinguir 4 tipos de riscos: para a criança, para a mãe, para o ambiente e para a sociedade.

Avaliar o efeito da alimentação com leite artificial na saúde comporta dificuldades técnicas. Para começar, as patologias estudadas têm, frequentemente, causas múltiplas, o que exige estudos com um «n» elevado e com alta qualidade estatística. Esta última é difícil de alcançar e, sobretudo por razões de carácter ético, não é possível fazer estudos randomizados. A grande maioria dos estudos são, por isso, observacionais, muitas vezes cross-sectional e, quando retrospectivos, sujeitos ao «viés da memória» (recall bias). As definições do que é amamentação, sobretudo do que é amamentação exclusiva, são variáveis entre os estudos. Além disso, nalgumas patologias há a hipótese de poder bastar uma dose de leite artificial para alterar os resultados (sensibilização às proteínas do leite de vaca, por exemplo).

Outra questão estatística é que quando não se prova uma associação entre dois factores, isso não significa que se tenha provado que não estão associados. Conseguir provar que dois factores não estão de facto e seguramente associados exige estudos caros e de enormes dimensões que raramente podem ser realizados.

\section{ALIMENTAÇÃO COM LEITE ARTIFICIAL: RISCOS PARA A CRIANÇA}

Apesar de todas as melhorias introduzidas nos últimos anos no leite artificial, a sua composição continua a ser totalmente diferente da do leite materno, que é um alimento «vivo»e se adapta às necessidades do bebé. A investigação continua a descobrir no leite materno factores envolvidos na protecção contra as infecções, no desenvolvimento da imunidade, etc. ${ }^{6}$ Para além disso, o acto de dar de mamar tem consequências fisiológicas benéficas, como, por exemplo, a segregação de hormonas na mãe envolvidas na vinculação mãe-bebé ou o desenvolvimento óptimo da cavidade bucal do bebé.

De tudo o que acima se expôs deduz-se que alimentar a criança com leite artificial comporta riscos.

Para começar, há um maior risco de mortalidade da criança. ${ }^{7,8}$ Se este facto é fácil de explicar em relação aos países subdesenvolvidos, já não é tão óbvio em relação a países desenvolvidos. No entanto, o estudo de Chen mostrou que, nos Estados Unidos da América ,o facto da criança nunca ter sido amamentada aumentava o risco de morte até ao ano de idade em $21 \%$. Mais do que a utilização em larga escala da vacina do Haemophilus influenzae ou do que melhores condições de higiene nos partos, a estratégia que, em maior medida, diminuiria a mortalidade infantil mundial, seria amamentar. $^{9}$

É consensualmente reconhecido e é uma das razões para a recomendação de amamentar exclusivamente durante 6 meses, o aumento do risco de gastroenterites agudas nos bebés alimentados com leite artificial. Esse risco estima-se em cerca de $40 \%$ a $65 \%$ no primeiro ano de vida. ${ }^{10,11}$

Também o risco de otite está bem estudado e não há controvérsia: este está aumentado em $50 \%$ nos bebés alimentados apenas com leite artificial, comparativamente aos bebés amamentados exclusivamente durante 4 meses ou mais. Este risco mantém-se durante, pelo menos, o $1^{\circ}$ ano de vida. ${ }^{12}$

Nos países desenvolvidos, os bebés alimentados com leite artificial têm, em média, mais $73 \%$ de risco de serem hospitalizados por infecção respiratória baixa. ${ }^{13}$ Com o aleitamento materno a protecção mantém-se até, pelo menos, aos 5 anos. $^{14}$

Em relação à asma e à atopia (incluindo a dermatite atópica), há controvérsia, talvez pela dificuldade em encontrar um grupo suficientemente vasto com uma alimentação congruente. Como antes se disse, há a hipótese de bastar uma toma de leite artificial para sensibilizar o bebé às proteínas do leite de vaca, alterando a protecção dada pelo leite materno. Seleccionando os estudos com melhor qualidade, demonstrou-se maior risco de atopia e asma na infância para as crianças alimentadas com leite artificial. ${ }^{15}$ Nos bebés amamentados, com história familiar de asma, há uma redução de 
$50 \%$ do risco. ${ }^{16,17}$ Há, ainda, um estudo, recentemente publicado na Thorax, que demonstrou que, aos 10 anos, crianças que nunca foram amamentadas têm uma pior função respiratória. ${ }^{18}$

Em relação à doença celíaca, há total concordância nos diversos estudos: introduzir o glúten durante a amamentação diminui o risco de desenvolver esta doença. ${ }^{19}$ Recomenda-se, por isso, introduzir o glúten depois dos 4 meses, durante a amamentação, mantendo esta última por algum tempo (idealmente até aos 2 anos ou mais, como recomendado pela OMS).

Em vários estudos e em algumas meta-análises foi demonstrado um aumento de 19 a $23 \%$ do risco de desenvolver diabetes mellitus tipo 1 nos bebés que nunca foram amamentados. ${ }^{20}$

Uma meta-análise de 2004 mostrou um aumento do risco de doença de Crohn de $33 \%$ e de colite ulcerosa, de $23 \%$, nos bebés alimentados com leite artificial. ${ }^{21}$ Há, no entanto, alguma controvérsia, sobretudo porque há uma predisposição genética para as doenças inflamatórias do intestino que, se não for tida em consideração, pode confundir os resultados. ${ }^{22,23}$ As crianças alimentadas com leite artificial têm, também, um maior risco (cerca de 15\%) de desenvolver leucémias agudas. O efeito é menos dramático em relação aos linfomas, embora pareça haver, também neste caso, um aumento do risco de desenvolver a doença. ${ }^{24}$

Em relação à obesidade, tanto infantil como no adulto, há relação entre o facto de não se ter sido amamentado e de se ter excesso de peso. Este efeito é dose-dependente (quanto mais tempo se amamenta maior o efeito protector), mas discreto, até porque a obesidade é uma doença multi-factorial. Constatou-se, portanto, que as pessoas não amamentadas têm, em média, índices de massa corporal (IMC) mais elevados..$^{25}$

Ser alimentado com leite artificial aumenta o risco de diabetes mellitus tipo 2 , o que está relacionado também com o aumento do IMC. ${ }^{26}$

Numa meta-análise e revisão sistemática encontraram-se valores discretamente mais altos de tensão arterial e de colesterol nos adultos que não foram amamentados. Apesar disso, o autor conclui que, nos E.U.A., esta diferença implica um aumento em $17 \%$ da hipertensão arterial, em $6 \%$ da doença coronária e em $15 \%$ de acidentes vasculares cerebrais e acidentes isquémicos transitórios. ${ }^{27}$
Em relação ao desenvolvimento cognitivo, não há ainda consenso. Alguns estudos demonstram um menor desenvolvimento cognitivo nas crianças alimentadas com leite artificial. No entanto, quando os resultados foram corrigidos para a inteligência materna, esse efeito era menor do que o inicialmente encontrado. ${ }^{28}$ Vários estudos, um dos quais deste ano, sublinham o aumento do risco de morte súbita do lactente (SIDS) quando o bebé é alimentado com leite artificial. ${ }^{29}$ Esse aumento é estimado entre 30 e $50 \%$, consoante os estudos. ${ }^{30}$ À luz destes dados, não se pode deixar de alertar para a possibilidade de a utilização de chupeta interferir no sucesso da amamentação. A chupeta foi, recentemente, incluída nas recomendações para a prevenção da SIDS, o que tem sido criticado por várias especialistas ligados à área do aleitamento materno. ${ }^{31}$

Um dos problemas associados à sucção no biberão ou na chupeta é que esta não é fisiológica, e pode promover a má oclusão dentária e as alterações da fonação, como demonstrado nalguns estudos. ${ }^{32}$

Num interessante estudo recentemente publicado na Pediatrics, recolheram-se informações detalhadas, de forma prospectiva, em 5.890 díades. Neste grupo, houve 512 relatórios de maus-tratos à criança. Constatou-se que a alimentação com leite artificial estava significativamente relacionada com negligência e violência materna, sobretudo com a primeira. A alimentação de qualquer tipo não tinha relação com os maus-tratos não-maternos. ${ }^{33}$

A contaminação do leite artificial é um risco real. Para além do caso recente da melamina presente no leite artificial chinês, e de se terem encontrado vestígios de melamina e ácido cianídrico em leites comercializados nos E.U.A, ${ }^{34}$ há referências a mortes neonatais devidas a infecções a Enterobacter sakazakii cuja fonte era o leite artificial. ${ }^{35,36}$ Regularmente há surtos de Salmonela, os últimos dos quais em Espanha e em França, ${ }^{37}$ de que resultaram mortes neonatais. De facto, o leite artificial em pó não é estéril mas apenas pasteurizado. É importante alertar as mães de recém-nascidos que optem por os alimentar com leite artificial para a importância de preparar o biberão e administrá-lo com alguma celeridade, para evitar a multiplicação destas bactérias.

Evidenciou-se que a exposição da grávida a poluentes, tais como os bifenilos policlorinados, durante o pe- 
ríodo de gestação, afecta o desenvolvimento neurológico infantil. Constatou-se que nas crianças amamentadas havia melhor recuperação neurológica do que nas crianças alimentadas a leite artificial. ${ }^{38,39}$

Uma criança não amamentada tem, ainda, outros riscos: por exemplo, não tem a possibilidade de beneficiar do efeito analgésico do leite materno, tão útil durante procedimentos tais como a vacinação, a colheita de sangue, ou mesmo durante a observação clínica. ${ }^{40}$ Há alguns pequenos estudos interessantes, mas que necessitam de ulteriores confirmações, tais como o de Campanozzi e colaboradores, que mostrou uma mais lenta resolução do refluxo gastro-esofágico nos bebés não-amamentados ${ }^{41}$ ou o de Nichols e colaboradores, que encontrou um aumento do risco de cancro da mama nas mulheres que não foram amamentadas em criança. $^{42}$

\section{ALIMENTAÇÃO COM LEITE ARTIFICIAL: RISCOS PARA A MÃE}

A utilização de leite artificial não tem só consequências negativas para as crianças. A amamentação é um acto fisiológico, portanto, parece plausível que não o fazer comporte algum risco também para a mãe. Esta hipótese é confirmada em diversos estudos.

Uma das razões para recomendar às mulheres que amamentem 2 anos ou mais é o aumento do risco de cancro da mama para quem não o faz. Nas mulheres em idade fértil esse risco reduz-se em $4 \%$ por cada ano de amamentação. ${ }^{43}$

Há também um aumento do risco de cancro do ovário e do endométrio. Quanto menor a duração da amamentação e quanto menos filhos tem, maior é o risco para a mulher de desenvolver cancro do ovário (esse aumento estima-se em 21\%). ${ }^{44-46}$

Em relação à obesidade, há associação entre não amamentar e o excesso de peso. Este facto explica-se porque nas mulheres que amamentam há alterações do metabolismo das gorduras e dos carbo-hidratos (que resultam também, num melhor controle da glicémia nas mulheres diabéticas que amamentam). ${ }^{47}$

Num estudo recente, foi analisada uma base de dados com 89.326 mulheres, enfermeiras do National Health Service britânico. Registaram-se 2.540 enfartes agudos do miocárdio (EAM). Sem correcção para outros factores de risco, houve diminuição de $37 \%$ do risco de ter um EAM nas mulheres que amamentaram 2 anos ou mais (cumulativo). Com correcção para outros factores de risco cardiovascular, a diminuição do risco era de $23 \%{ }^{48}$

Em relação à depressão pós-parto, tem havido alguma dificuldade em perceber se é o facto de a mãe estar deprimida que dificulta a amamentação ou se, pelo contrário, é não dar de mamar que a predispõe para a depressão. Há estudos que provam ambas as hipóteses. ${ }^{49,50} \mathrm{Em}$ termos fisiológicos, a acção hormonal da prolactina e da oxitocina atenuam os sintomas da depressão. De facto, parece haver um maior nível de stress e angústia nas mães que não amamentam. ${ }^{51}$

Em relação à diabetes mellitus tipo 2 constatou-se que, nas mães que não tiveram diabetes gestacional, não amamentar comporta um risco de $12 \%$ de desenvolver a doença, enquanto que nas mães que amamentam esse risco é de apenas $4 \%{ }^{52,53}$

Um estudo recente avaliou, numa base de dados britânica com 1.289.029 mulheres, a relação entre doença da vesícula biliar na meia-idade e a vida reprodutiva. Chegou-se à conclusão que, por cada parto, há um aumento do risco de $8 \%$, mas por cada ano de amamentação há uma diminuição do risco de $7 \%$. As autoras concluem que ter filhos aumenta o risco de doença das vias biliares, mas que este risco pode ser eliminado através da amamentação. ${ }^{54}$

Em relação à osteoporose, há o famoso estudo de Karlsson e colaboradores em que se demonstrou que, durante a amamentação, há uma discreta osteopénia, mas que, 6 meses após o desmame, a massa óssea da mulher é superior à determinada antes da gravidez. Daqui se pode concluir que não amamentar aumenta o risco de osteoporose. ${ }^{55}$

É sempre bom realçar que não amamentar comporta um risco maior de engravidar de novo. ${ }^{56} \mathrm{~A}$ amamentação parece ser um processo fisiológico de espaçamento das gravidezes. Este facto é confirmado pela eficácia do Método da Amenorreia Lactacional (Programa Nacional de Saúde Reprodutiva 2008).

\section{ALIMENTAÇÃO COM LEITE ARTIFICIAL: RISCOS PARA O AMBIENTE}

A alimentação dos filhos com leite artificial comporta diversos riscos também para o ambiente. Enquanto que o acto de dar de mamar não requer qualquer tipo de ob- 
jecto, a alimentação com leite artificial exige uma série de utensílios, tais como biberões, esterilizadores, tetinas, etc., feitos de materiais (plástico, silicone, metal) não-biodegradáveis. Estes têm efeitos negativos porque, para a sua produção, são gastos materiais e recursos que começam a escassear, e porque, uma vez acabada a sua vida útil, se acumulam no meio ambiente, com tempos de degradação prolongados.

Não podemos esquecer que a produção do leite em si é altamente poluente. A criação de gado implica o cultivo intensivo dos campos para satisfazer as necessidades alimentares dos animais, a utilização de fármacos que são eliminados nas águas, e a produção elevada de metano. Todos estes factores sobrecarregam o ambiente e afectam os ecosistemas.

A propósito de materiais biodegradáveis, é bom recordar que uma mulher que amamenta até aos 2 anos ou mais tem, frequentemente, amenorreia, que pode durar muitos meses. Portanto, nesse período, não utilizará pensos higiénicos, contribuindo, assim, para a redução do impacto negativo da acumulação destes no ambiente.

É claro que estas não serão as principais razões que levarão uma mãe a evitar o leite artificial, mas num momento em que a ecologia e a defesa do ambiente se revelam fundamentais para a preservação do nosso planeta, sublinhar as repercussões negativas para o ambiente da alimentação com leite artificial torna-se eticamente necessário. ${ }^{57}$

\section{ALIMENTAÇÃO COM LEITE ARTIFICIAL: RISCOS PARA A SOCIEDADE}

Alimentar um bebé com leite artificial comporta elevados custos para a família, desde a compra dos «consumíveis» como biberões, tetinas e afins, até ao preço do próprio leite. Numa altura de crise económica, a enorme poupança que a amamentação permite não é desprezível.

Como já vimos, a escolha de não amamentar tem consequências sobretudo em relação ao risco de vir a sofrer de uma série de doenças agudas e crónicas. Estas terão, seguramente, efeitos negativos a nível orçamental, não só no orçamento das famílias, como no orçamento do Estado.

Para além disso, alguns estudos sugerem que dar de mamar é um factor psicoprotector para a criança. ${ }^{58}$ É bem conhecida na Psicologia do Desenvolvimento a importância da vinculação mãe-bebé para a saúde mental do indivíduo. ${ }^{59}$ Durante a amamentação é segregada a oxitocina, conhecida como a «hormona do amor», que facilita a vinculação. ${ }^{60} \mathrm{O}$ facto da mulher não amamentar o seu bebé poderá, então, estar relacionado com um maior sofrimento emocional para o futuro adulto, que o poderão levar a condutas anti-sociais, com custos pesados para o indivíduo e para a sociedade.

\section{O ALEITAMENTO MATERNO EM PORTUGAL}

Se há, então, provas tão abrangentes dos riscos inerentes à alimentação com leite artificial, porque é que, segundo a OMS, apenas $1 \mathrm{em} 3$ mulheres ainda amamenta aos 6 meses?

Em Portugal os dados oficiais são antigos (2002), e têm falhas técnicas. Um estudo recente, ${ }^{61}$ conduzido na maternidade do Hospital Santa Maria, mostra que 97\% das mulheres amamentam à saída da Maternidade; $54,7 \%$ aos 3 meses e $34,1 \%$ aos 6 meses, o que vem confirmar os dados da OMS. No entanto, é a própria OMS que, em vários documentos, afirma que mais de $98 \%$ das mulheres pode amamentar. E é fisiológico o facto que qualquer mulher que tenha estado grávida possa induzir a lactação.

Então porque é que as taxas de amamentação são tão baixas?

Há vasta investigação sobre esse assunto. Podemos sublinhar os factores mais importantes: a alimentação das crianças com alimentos confeccionados, sejam eles leite artificial, sejam papas, boiões, biscoitos, etc., é um comércio em expansão que mobiliza muito dinheiro. Apesar do já referido Código de Ética do Marketing dos Substitutos do Leite Materno, ainda é feita muita pressão junto aos profissionais de saúde e aos cidadãos para que acreditem que a utilização de tais produtos é indispensável para o crescimento infantil.

A nossa sociedade valoriza muito a produtividade que tenha um retorno monetário. Ser mãe é uma actividade não remunerada e, por isso, culturalmente desvalorizada. O impacto das ideias sociais e culturais sobre a maternidade e amamentação são de extrema importância.

De facto, vivemos numa «cultura do biberão», onde tudo e todos levam as mães a duvidarem da própria ca- 
pacidade de amamentar e a procurar nos profissionais de saúde, nos técnicos sociais, nos professores, nos livros, em cursos, as respostas para as suas dúvidas. Os profissionais não têm, geralmente, os conhecimentos necessários para apoiá-las, e dão, com frequência, indicações que dificultam ou inviabilizam a amamentação, acabando por diminuir a confiança das mães na própria capacidade de alimentar os seus filhos. Assim, as mães sentem-se de tal modo fragilizadas no seu papel que abdicam do próprio instinto maternal e necessitam de ler no biberão quantos mililitros de leite os seus filhos estão a ingerir para não ficarem ansiosas com o medo de não conseguirem nutri-los suficientemente.

Seria, por isso, importante que a formação dos profissionais de saúde valorizasse a promoção, protecção e apoio do aleitamento materno.

\section{CONCLUSÃO}

É claro então que, enquanto cidadãos e enquanto profissionais de saúde, podemos e temos a obrigação de promover, proteger e apoiar o aleitamento materno. Devemos fazê-lo com respeito pela decisão de cada mãe, sem culpabilizar a escolha de não amamentar. A mãe é a verdadeira especialista do seu próprio filho.

Apoiar o aleitamento materno não é uma questão de fundamentalismo, retorno ao passado ou falta de modernidade, mas é uma questão de honestidade científica e de ecologia social e intelectual.

\section{REFERÊNCIAS BIBLIOGRÁFICA}

1. Cattaneo A, Yngve A, Koletzko B, Guzman LR; Promotion of Breastfeeding in Europe project. Protection, promotion and support of breastfeeding in Europe: current situation. Public Health Nutr 2005 Feb; 8(1): 39-46.

2. Infant and young child feeding: standard recommendations for the European Union. Disponível em: http://www.burlo.trieste.it/documenti/EUpolicy06en.pdf [acedido em 23/06/2009].

3. Gartner LM, Morton J, Lawrence RA, Naylor AJ, O'Hare D, Schanler RJ, et al. Breastfeeding and the use of human milk. Pediatrics 2005 Feb; 115 (2): 496-506.

4. World Health Organization. Promoting proper feeding for infants and young children. Disponível em: http://www.who.int/nutrition/topics/infantfeeding/en/ [acedido em 23/06/2009].

5. Direcção-Geral de Saúde. Código Internacional de Marketing de Substitutos do Leite Materno. Lisboa: DGS; 2008. p. 12.

6. Rautava S, Walker WA. Academy of Breastfeeding Medicine Founder's Lecture 2008: Breastfeeding: an extrauterine link between mother and child. Breastfeeding Me 2009 Mar; 4 (1): 3-10.
7. Chen A, Rogan WJ. Breastfeeding and the risk of postneonatal death in the United States. Pediatrics 2004 May;113(5):e435-e9.

8. Edmond KM, Zandoh C, Quigley MA, Amenga-Etego S, Owusu-Agyei $S$, Kirkwood BR. Delayed breastfeeding initiation increases risk of neonatal mortality. Pediatrics 2006 Mar; 117 (3): e380-6.

9. Claeson M, Gillespie D, Mshinda H, Troedsson H, Victora CG; Bellagio Study Group on Child Survival. Knowledge into action for child survival. Lancet 2003 Jul 26; 362 (9380): 323-7.

10. Quigley MA, Kelly YJ, Sacker A. Infant feeding, solid foods and hospitalisation in the first 8 months after birth.Arch Dis Child 2009 Feb; 94 (2): 148-50.

11. Chien PF, Howie PW. Breast milk and the risk of opportunistic infection in infancy in industrialized and nonindustrialized settings. Adv Nutr Res 2001; 10: 69-104.

12. Duffy LC, Faden H, Wasielewski R, Wolf J, Krystofik D. Exclusive breastfeeding protects against bacterial colonization and day care exposure to otitis media. Pediatrics 1997 Oct; 100 (4): E7.

13. Bachrach VR, Schwarz E, Bachrach LR. Breastfeeding and the risk of hospitalization for respiratory disease in infancy: a meta-analysis. Arch Pediatr Adolesc Med 2003 Mar; 157 (3): 237-43.

14. Broor S, Pandey RM, Ghosh M, Maitreyi RS, Lodha R, Singhal T, et al. Risk factors for severe acute lower respiratory tract infection in underfive children. Indian Pediatr 2001 Dec; 38 (12): 1361-9.

15. Oddy WH. The long-term effects of breastfeeding on asthma and atopic disease Adv Exp Med Biol 2009; 639: 237-51.

16. Hatakka K, Piirainen L, Pohjavuori S, Poussa T, Savilahti E, et al. Allergy in day care children: prevalence and environmental risk factors.Acta Paediatr 2009 May; 98 (5): 817-22.

17. Oddy WH, Peat JK. Breastfeeding, asthma and atopic disease: an epidemiological review of literature. J Hum Lact 2003 Aug; 19 (3): 250$-61$.

18. Ogbuanu IU, Karmaus W, Arshad SH, Kurukulaaratchy RJ, Ewart S. Effect of breastfeeding duration on lung function at age 10 years: a prospective birth cohort study Thorax 2009 Jan; 64 (1): 62-6.

19. Agostoni C, Shamir R. Can a change in policy of complementary infant feeding reduce the risk for type 1 diabetes and celiac disease? Pediatr Endocrinol Rev 2008 Sep; 6 (1): 2-4.

20. Rosenbauer J, Herzig P, Giani G. Early infant feeding and risk of type 1 diabetes mellitus: a nationwide population-based case-control study in pre-school children. Diabetes Metab Res Rev 2008 Mar-Apr; 24 (3): 211-22.

21. Klement E, Cohen RV, Boxman J, Joseph A, Reif S. Breastfeeding and risk of inflammatory bowel disease: a systematic review with meta-analysis. Am J Clin Nutr 2004 Nov; 80 (5): 1342-52.

22. Barclay AR, Russell RK, Wilson ML, Gilmour WH, Satsanji J, Wilson DC. Systematic review: the role of breastfeeding in the development of pediatric inflammatory bowel disease. J Pediatr 2009 May 21 [article in press: doi:10.1016/j.jpeds.2009.03.017].

23. Mikhailov TA, Furner SE. Breastfeeding and genetic factors in the etiology of inflammatory bowel disease in children. World J Gastroenterol 2009 Jan 21; 15 (3): 270-9.

24. Zur Hausen $\mathrm{H}$. Childhood leukemias and other hematopoietic malignancies: Interdependence between an infectious event and chromosomal modifications. Int J Cancer. 2009 Feb 18. [article in press: doi: 


\subsection{2/ijc.24365]}

25. Harder T, Bergmann R, Kallischnigg G, Plagemann A. Duration of breastfeeding and risk of overweight: a meta-analysis. Am J Epidemiol 2005 Sep 1; 162 (5): 397-403.

26. Mayer-Davis EJ. Type 2 diabetes in youth: epidemiology and current research toward prevention and treatment.J Am Diet Assoc 2008 Apr; 108 (4 Suppl 1): S45-51.

27. Martin RM, Smith GD, Mangtani P, Tilling K, Frankel S, Gunnell D.. Breastfeeding and cardiovascular mortality: the Boyd Orr cohort and a systematic review with meta-analysis. Eur Heart J 2004 May; 25 (9): 778-86.

28. Der G, Batty GD, Deary IJ. Effect of breast feeding on intelligence in children: prospective study, sibling pairs analysis, and meta-analysis. BM] 2006 Nov 4; 333 (7575): 945.

29. Vennemann MM, Findeisen M, Butterfass-Bahloul T, Jorch G, Brinkmann B, Köpcke W, et al. Modifiable risk factors for SIDS in Germany: results of GeSID. Acta Paediatr 2005 Jun; 94 (6): 655-60

30. Vennemann MM, Findeisen M, Butterfass-Bahloul T, Jorch G, Brinkmann B, Köpcke W, et al. Modifiable risk factors for SIDS in Germany: results of GeSID. Acta Paediatr 2005 Jun; 94 (6): 655-60.

31. Stuebe A, Lee K. The pacifier debate. Pediatrics 2006 May; 117 (5): $1848-$ 9; author reply 1850-3.

32. Viggiano D, Fasano D, Monaco G, Strohmenger L. Breast feeding, bottle feeding, and non-nutritive sucking; effects on occlusion in deciduous dentition. Arch Dis Child 2004 Dec; 89 (12): 1121-3.

33. Strathearn L, Mamun AA, Najman JM, O'Callaghan MJ. Does breastfeeding protect against substantiated child abuse and neglect? A 15year cohort study. Pediatrics 2009 Feb; 123 (2): 483-93.

34. Turnipseed S, Casey C, Nochetto C, Heller DN. Determination of Melamine and Cyanuric Acid Residues in Infant Formula using LCMS/MS U.S. FDA Laboratory Information Bulletin n. ${ }^{\circ}$ 4421, 2008 Oct; Vol. 24.

35. Weir E. Powdered infant formula and fatal infection with Enterobacter sakazakii. CMAJ 2002 Jun 11; 166 (12):1570.

36. van Acker J, de Smet F, Muyldermans G, Bougatef A, Naessens A, Lauwers S. Outbreak of necrotizing enterocolitis associated with Enterobacter sakazakii in powdered milk formula. J Clin Microbiol 2001 Jan; 39 (1): 293-7.

37. Jourdan N, Le Hello S, Delmas G, Clouzeau J, Manteau C, Desaubliaux $B$, et al. Nationwide outbreak of Salmonella enterica serotype gives infections in infants in France, linked to infant milk formula, September 2008. Euro Surveill 2008 Sep 25;13 (39). pii: 18994.

38. Arendt V. Communicating human biomonitoring results to ensure policy coherence with public health recommendations: analysing breastmilk whilst protecting, promoting and supporting breastfeeding. Environ Health 2008 Jun 5;7 Suppl 1: S6.

39. Vreugdenhil HJ, Van Zanten GA, Brocaar MP, Mulder PG, Weisglas-Kuperus N. Prenatal exposure to polychlorinated biphenyls and breastfeeding: opposing effects on auditory P300 latencies in 9-year old Dutch children. Dev Med Child Neurol 2004 Jun; 46 (6): 398-405.

40. Shah PS, Aliwalas L, Shah V. Breastfeeding or breastmilk to alleviate procedural pain in neonates: a systematic review. Breastfeed Med 2007 Jun; 2 (2): 74-82.

41. Campanozzi A, Boccia G, Pensabene L, Panetta F, Marseglia A, Strisciu- glio $P$, et al. Prevalence and natural history of gastroesophageal reflux: pediatric prospective survey. Pediatrics 2009 Mar; 123 (3): 779$-83$.

42. Nichols HB, Trentham-Dietz A, Sprague BL, Hampton JM, Titus-Ernstoff $L$, Newcomb PA. Effects of birth order and maternal age on breast cancer risk: modification by whether women had been breast-fed.Epidemiology 2008 May;19 (3): 417-23.

43. Kwan ML, Kushi LH, Weltzien E, Maring B, Kutner SE, Fulton RS, et al. Epidemiology of breast cancer subtypes in two prospective cohort studies of breast cancer survivors. Breast Cancer Res 2009 May 22; 11 (3): R31.

44. Okamura C, Tsubono Y, Ito K, Niikura H, Takano T, Nagase S, et al. Lactation and risk of endometrial cancer in Japan: a case-control study. Tohoku J Exp Med 2006 Feb; 208 (2): 109-15.

45. Tung KH, Goodman MT, Wu AH, McDuffie K, Wilkens LR, Kolonel LN, et al. Reproductive factors and epithelial ovarian cancer risk by histologic type: a multiethnic case-control study. Am J Epidemiol 2003 Oct 1; 158 (7): 629-38.

46. Tung KH, Wilkens LR, Wu AH, McDuffie K, Nomura AM, Kolonel LN, et al. Effect of anovulation factors on pre- and postmenopausal ovarian cancer risk: revisiting the incessant ovulation hypothesis. Am J Epidemiol 2005 Feb 15;161 (4): 321-9.

47. Walker L, Freeland-Graves JH, Milani T, George G, Hanss-Nuss H, Kim $M$, et al. Weight and behavioral and psychosocial factors among ethnically diverse, low-income women after childbirth: II. Trends and correlates. Women Health 2004; 40 (2): 19-34.

48. Stuebe AM, Michels KB, WillettWC, Manson JE, Rexrode K, Rich-Edwards $J W$. Duration of lactation and incidence of myocardial infarction in middle to late adulthood Am J Obstet Gynecol. 2009 Feb; 200(2):138.e1-8.

49. Seimyr L, Edhborg M, Lundh W, Sjögren B. In the shadow of maternal depressed mood: experiences of parenthood during the first year after childbirth. J Psychosom Obstet Gynecol 2004 Mar;25 (1): 23-34.

50. Henderson JJ, Evans SF, Straton JA, et al. Impact of postnatal depression on breastfeeding duration. [erratum appears in Birth. 2004 Mar; 31 (1): 76]. Birth 2003 Sep; 30 (3): 175-80.

51. Groër MW. Differences between exclusive breastfeeders, formula-feeders, and controls: a study of stress, mood, and endocrine variables. Biol Res Nurs 2005 Oct; 7 (2): 106-17.

52. Villegas R, Gao YT, Yang G, Li HL, Elasy T, Zheng W, et al. Duration of breast-feeding and the incidence of type 2 diabetes mellitus in the Shanghai Women's Health Study. Diabetologia 2008 Feb; 51 (2): 258-66 .

53. Stuebe AM, Rich-Edwards JW, Willett WC, Manson JE, Michels KB. Duration of lactation and incidence of type 2 diabetes. JAMA 2005 Nov 23;294 (20): 2601-10.

54. Liu B, Beral V, Balkwill A; Million Women Study Collaborators. Childbearing, breastfeeding, other reproductive factors and the subsequent risk of hospitalization for gallbladder disease. Int J Epidemiol 2009 Feb; 38 (1): 312-8.

55. Karlsson MK, Ahlborg HG, Karlsson C. Maternity and mineral density Acta Orthop 2005 Feb; 76 (1): 2-13.

56. Egbuonu I, Ezechukwu CC, Chukwuka JO, Ikechebelu JI. Breast-feeding, return of menses, sexual activity and contraceptive practices among 
mothers in the first six months of lactation in Onitsha, South Eastern Nigeria.J Obstet Gynaecol 2005 Jul; 25 (5): 500-3.

57. Radford A. The ecological impact of bottle feeding. Cambridge: Baby Milk Action; 1991.

58. Schepker R. Beitrage aus der Kinder- und Jugendpsychiatrie zur Pravention und Integration bei Kindern in Zuwandererfamilien. Prax Kinderpsychol Kinderpsychiatr 2009; 58 (4): 263-77.

59. Bretherton I. The origins of attachment theory: John Bowlby and Mary Ainsworth. Dev Psychol 1992 Sep; 28 (5): 759-75.

60. Klaus M. Mother and infant: early emotional ties. Pediatrics 1998 Nov;
102 (5 Suppl E):1244-6.

61. Sandes AR, Nascimento C, Figueira J, Gouveia R, Valente S, Martins S, et al.Aleitamento materno: prevalência e factores condicionantes. Acta Med Port 2007 Maio-Jun; 20 (3): 193-200.

Conflitos de Interesse: não assinalados

\section{ENDEREÇO PARA CORRESPONDÊNCIA}

Mónica Pina

E-mail: liga.do.leite@gmail.com

\section{ABSTRACT}

In this article we review the risk associated with feeding children with formula, specifically the risks to the child, the mother, the environment and the society. For the child there is a higher risk of, among others, mortality, gastrointestinal infection, otitis, lower respiratory infection, asthma and atopy, celiac disease, diabetes mellitus type 1 , inflammatory bowel disease, acute leukemia and lymphoma, obesity, diabetes mellitus type 2, arterial hypertension and cholesterol, sudden infant death and dental malocclusion. For the mother there is a higher risk of, among others, breast cancer, ovarian cancer, obesity, acute myocardial infarction, diabetes mellitus type 2, post-partum depression, biliary disease and osteoporosis. For the environment, feeding children with formula raises the use of resources that are decreasing, and the accumulation of non-biodegradable waste. For society, there are consequences on the famlily and the state bill, and consequences related with the possibility of altered mother-child bonding.

Our aim was to reinforce the importance of promoting, protecting and supporting breastfeeding.

Keywords: Breastfeeding; Infant Formula; Risk. 